University of Nebraska - Lincoln

DigitalCommons@University of Nebraska - Lincoln

P. F. (Paul Frazer) Williams Publications

Electrical \& Computer Engineering, Department

February 1985

\title{
Numerical simulation of streamer propagation in nitrogen at atmospheric pressure
}

\author{
S. K. Dhali \\ Texas Tech University, Lubbock, TX \\ P. F. Williams \\ University of Nebraska - Lincoln, pfw@moi.unl.edu
}

Follow this and additional works at: https://digitalcommons.unl.edu/elecengwilliams

Part of the Electrical and Computer Engineering Commons

Dhali, S. K. and Williams, P. F., "Numerical simulation of streamer propagation in nitrogen at atmospheric pressure" (1985). P. F. (Paul Frazer) Williams Publications. 26.

https://digitalcommons.unl.edu/elecengwilliams/26

This Article is brought to you for free and open access by the Electrical \& Computer Engineering, Department of at DigitalCommons@University of Nebraska - Lincoln. It has been accepted for inclusion in P. F. (Paul Frazer) Williams Publications by an authorized administrator of DigitalCommons@University of Nebraska - Lincoln. 


\title{
Numerical simulation of streamer propagation in nitrogen at atmospheric pressure
}

\author{
S. K. Dhali* \\ Department of Electrical Engineering, Texas Tech University, \\ Lubbock, Texas 79409 \\ P. F. Williams ${ }^{\dagger}$ \\ Departments of Electrical Engineering and Physics, \\ Texas Tech University, Lubbock, Texas 79409
}

(Received 16 November 1984)

\begin{abstract}
Results of the application of flux-corrected transport techniques to a two-dimensional numerical simulation of streamer propagation are presented. Characteristics such as diameter, velocity, shape, and density gradient of the head, and ionization in the body of the propagating streamer are determined. These results provide new insight into streamer propagation.
\end{abstract}

Space-charge fields have long been known to play an important role in the over-volted breakdown of gases at atmospheric and higher pressure, due to the formation of domains of relatively high ionization density and low electric field. ${ }^{1-4}$. These collective phenomena, called streamers, are described by the solutions of the coupled transport and field equations under conditions for which they are highly nonlinear and difficult to solve without approximation. Most analytic approaches to solving these equations have assumed the solutions to be one dimensional. ${ }^{5-8}$ Such approximations ignore shape effects on the field, and may therefore significantly underestimate the peak field enhancement and badly overestimate the spatial range of the enhancement. Numerical approaches to the problem have generally made use of the method of characteristics to solve the one-dimensional transport equations, although in most reports the field was calculated in an approximately correct two-dimensional way. ${ }^{9-12}$ Conventional numerical techniques generally have difficulties in dealing with the very large density and field gradients which appear at the head of propagating streamers. ${ }^{13}$

We present here the results of numerical calculations of streamer propagation making use of two-dimensional fluxcorrected transport (FCT) techniques, which allow us to solve numerically the transport equations under strongly space-charge-dominated conditions such as those that occur at the head of propagating streamers. To our knowledge, our work represents the first systematic application of this technique to the problem of strongly space-chargedominated transport in a fully two-dimensional model, and our results are the first report of solutions of the transport equations under these conditions. FCT algorithms were originally developed to deal with the very steep density gradients that appear in shock wave solutions of the transport equations for fluids. ${ }^{14,15}$ Morrow and Lowke have applied these techniques to one-dimensional studies of spacecharge-dominated transport in gases. ${ }^{16}$ Fernsler has reported the use of a modified sharp and smooth transport algorithm (SHASTA) FCT algorithm ${ }^{14}$ to carry out twodimensional streamer simulations, but the work was not pursued..$^{17}$

For the streamer initiation we use as an initial condition a small, localized, neutral plasma of density substantially greater than the critical density for streamer formation, thereby by-passing the avalanche stage needed for single or few electron initiation conditions. Under the conditions we have investigated, we find the following: (1) quasi-steadystate, streamerlike solutions propagating with typical velocities of $1-3 \times 10^{8} \mathrm{~cm} / \mathrm{sec}$ exist; (2) in the quasi steady state, the properties of the streamer are independent of the density but not of the shape of the initiating plasma; (3) the ionization density in the body of a propagating streamer is a weak function of environmental parameters, approaching a steady-state value of $10^{14}-10^{15} \mathrm{~cm}^{-3}$, independent of the initiating density; (4) the dielectric relaxation time of the plasma at the streamer tip plays an important role in determining propagation velocity and ionization density in the streamer body; (5) the propagation velocity of both anode- and cathode-directed streamers is a function of several factors, including the electron density in front of the streamer and the shape of the streamer head. In the calculations we report here we find that the velocity and the electron density ahead of the streamer are related approximately logarithmically.

In the equilibrium hydrodynamic transport model, the electron and ion densities obey

$$
\begin{aligned}
\frac{\partial n_{e}}{\partial t}+\nabla \cdot\left(n_{e} \mathbf{v}_{e}\right) & =\alpha v_{e} n_{e}+\text { other terms, } \\
\frac{\partial n_{p}}{\partial t}+\nabla \cdot\left(n_{p} \mathbf{v}_{p}\right) & =\alpha v_{e} n_{e}+\text { other terms, }
\end{aligned}
$$

where $n_{e}$ and $n_{p}$ are the electron and positive ion densities, respectively, $v_{e}$ and $v_{p}$ are the corresponding drift velocities, $\alpha$ is the Townsend ionization coefficient, and the other terms may describe effects of diffusion or any of several particle source or sink mechanisms such as photoionization, attachment, or recombination. The drift velocities and $\alpha$ are functions of the local field which is given by $\nabla \cdot \mathbf{E}=\rho / \epsilon_{0}$, where $\rho=q_{e}\left(n_{p}-n_{e}\right)$ is the net charge density, $q_{e}$ being the (unsigned) electronic charge.

In our calculations a modified Euler finite difference approximation was used to carry out the time integration of Eqs. (1), and the low- and high-order fluxes were calculated according to donor cell and eighth-order difference schemes, respectively. The electric field was calculated with a novel algorithm based on Fourier transforming in the axial direc- 
tion and using a cubic spline for integration in the radial direction. ${ }^{18}$ The calculations were carried out using parameter values appropriate for nitrogen at 760 Torr, placed in a gap of $0.5 \mathrm{~cm}$ electrode separation. To conform to a set of experiments on laser-triggered breakdown, a load resistor of $50 \Omega$ was chosen, but for all simulations reported here, the voltage drop across the load was negligible. Parameters were $9,19 \quad \alpha / P=5.7 e^{-260 P / E}, \quad v_{e}=2.9 \times 10^{5} E / P, \quad v_{p}=2.0$ $\times 10^{3} E / P, D_{L}=1.8 \times 10^{3}$, and $D_{T}=2.19 \times 10^{3}$, where $P$ is in Torr, all lengths are in $\mathrm{cm}$, and $D_{L}$ and $D_{T}$ are the longitudinal and transverse diffusion coefficients, respectively. Although explicitly included, diffusive effects were negligible in all calculations discussed here. In order to by-pass the avalanche stage, we used initial conditions consisting of a small spheroid (ellipsoid of revolution) or hemispheroid of relatively dense neutral plasma, with Gaussian density distribution, placed either in the center of the gap or on one electrode.

The presence of free electrons ahead of the streamer tip, for which there are numerous sources, ${ }^{12}$ can strongly affect the streamer propagation. Except perhaps for drift in the case of anode-directed streamers, the detailed mechanism whereby these advance electrons are created is a secondary issue for streamer propagation. Accordingly, in the calculations described here, a weak, uniform neutral-background ionization was added as an initial condition, allowing study of the dependence of streamer propagation on the strength of the ionization ahead of it.

Figure 1 shows the electron density for several times for a calculation in which a plasma spheroid was placed initially in the center of gap. The pictures were made with the aid of a Comtal Vision One/20 image processing system, and the intensity at a point is proportional to the electron density at that point. Both anode- and cathode-directed streamers are seen in this figure. The anode-directed streamer starts

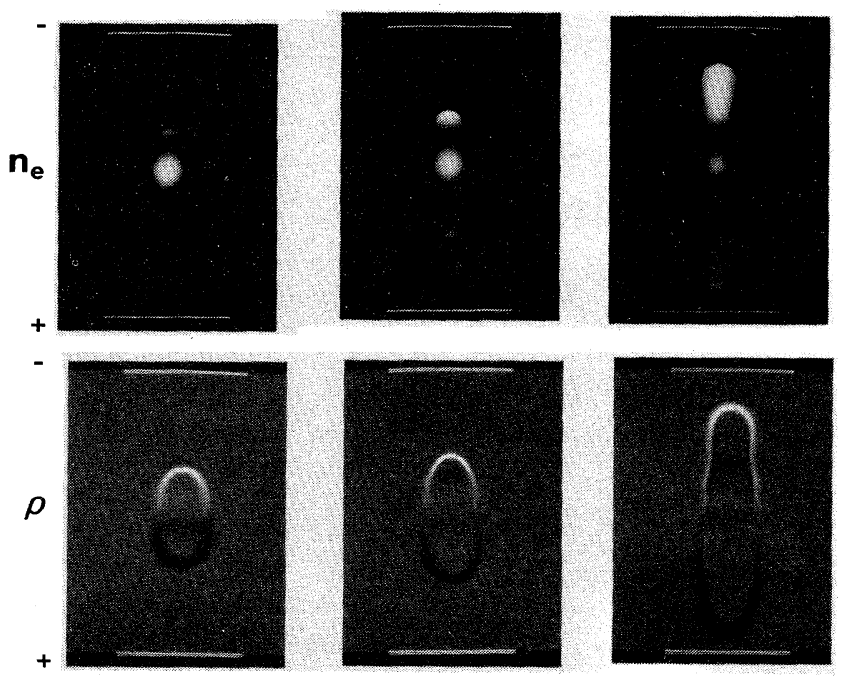

FIG. 1. Electron density $n_{e}$ and net charge density $\rho$, for $t=0.5$, 1.0 , and $1.25 \mathrm{~ns}$ for a calculation in which a Gaussian spheroid of plasma was initially placed in the center of the gap. The plasma density was $10^{14} \mathrm{~cm}^{-3}$ and the axial and transverse radii were 0.27 and $0.065 \mathrm{~mm}$, respectively. The gap spacing was $0.5 \mathrm{~cm}$, the voltage was $30 \mathrm{kV}$, making it about $70 \%$ overvolted, and there was a uniform background initial ionization density of $10^{8} \mathrm{~cm}^{-3}$. a)

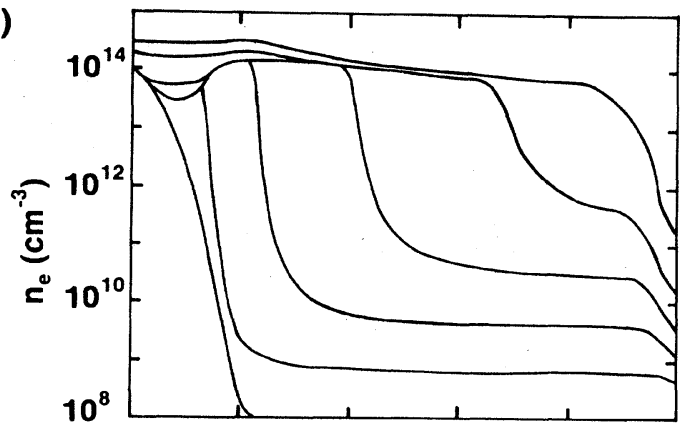

b)

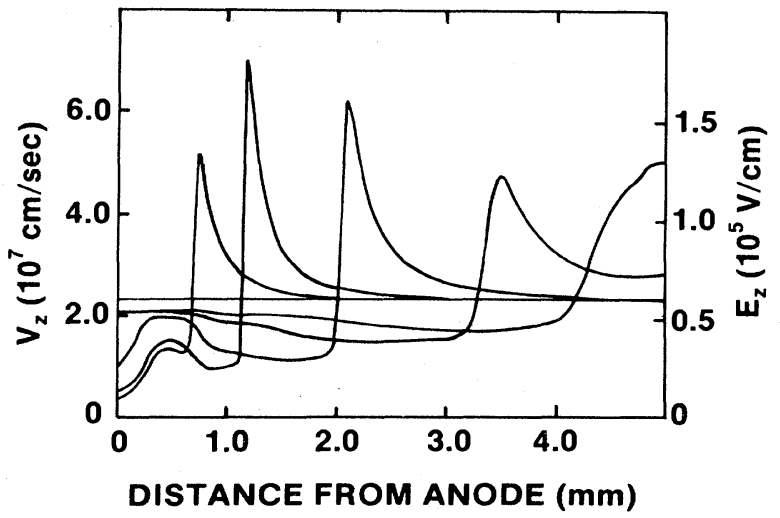

FIG. 2. On-axis electron density $n_{e}$ and axial electric field $E_{z}$, for $t=0.0,0.5,1.0,1.5,2.0$, and $2.5 \mathrm{~ns}$ for a cathode-directed streamer. The conditions were the same as in Fig. 1, except that the initiating plasma was a hemispheroid placed on the anode.

sooner, propagates faster, is a little broader, has a more shallow gradient at the tip, and leaves behind a smaller plasma density than the cathode-directed streamer.

Figure 2 shows the electron density and the axial component of the electric field for a cathode-directed streamer. The conditions were the same as in Fig. 1, except that the initiating plasma consisted of a hemispheroid placed on one electrode. After an initial period of adjustment, the streamer reaches nearly a steady-state propagation. The steady increase in background free-electron density is due to avalanche multiplication in the applied field. From the field plots, it is evident that the plasma inside the body of the streamer is not completely shielded from the external field, implying that the dielectric relaxation time $\tau_{d}$ is not negligible. For $n_{e}=10^{14} \mathrm{~cm}^{-3}, \tau_{d}=14 \mathrm{ps}$, comparable with the time required for the streamer to travel a distance equal to the width of the front. Our numerical results clearly show that the dielectric relaxation of the streamer plasma is far from complete, and call into question calculations such as those of Lozanskii ${ }^{20}$ in which a fully relaxed, metallic model for the streamer body is assumed.

In order to investigate the dependence of the streamer on the initiating plasma hemispheroid, additional calculations using differing initial conditions were carried out. In one set, the ionization density in the initial hemispheroid was $10^{15}$ instead of $10^{14} \mathrm{~cm}^{-3}$, but conditions were otherwise identical. It was found that once removed from the Gaussian tail of the initial plasma spheroid, the properties of the streamers were essentially the same in the two cases, implying that the streamer, once formed, is independent of the 
initial charge density. On the other hand, another set of calculations made with the same initial density, but with differing radii of the initial plasma hemispheroid, showed stable propagating streamers with radii varying with the radius of the initial spheroid. This observation is somewhat surprising since the field enhancement outside an oblong metallic object is strongly dependent on the sharpness of the tip. The fact that quasistable propagation can occur for a range of head shapes is probably a result of the incomplete shielding in the streamer body due to dynamic effects discussed above, and to the trade-off between maximum field enhancement and the range of this enhancement as a function of tip radius. The observation also suggests that quasitwo-dimensional calculations in which a reasonable head shape is arbitrarily imposed on the streamer ${ }^{9-12,20}$ may be more accurate than would otherwise be supposed.

The dependence of the progagation velocity, $V_{s}$, on the streamer environment is of interest. If we neglect the "other terms" in Eqs. (1) and assume a steady-state, axially propagating solution, then if $v_{e}<<V_{S}$,

$$
V_{S}=\frac{\int_{z_{1}}^{z_{2}} f(u) d u}{\ln \left(n_{2} / n_{1}\right)},
$$

where $\alpha v_{e}-\nabla \cdot \mathbf{v}_{e}=f\left(z-V_{S} t\right)$, and $n_{1}$ and $n_{2}$ are the electron densities at $z_{1}$ and $z_{2}$, respectively. If we choose $z_{1}$ and $z_{2}$ ahead and behind the streamer tip, then $n_{1}$ and $n_{2}$ are the densities ahead of and inside the streamer. Using the results of our numerical simulations, we find that Eq. (2) is well satisfied while the streamer is undergoing steady-state propagation.

Unfortunately the dependence of $V_{S}$ on $n_{1}$ in Eq. (2) is not explicit. Loeb ${ }^{21}$ has reported an approximate expression for $V_{S}$ which results from Eq. (2) by taking the integral to be constant and neglecting the $\nabla \cdot v_{e}$ term in $f(u)$. Fernsler, ${ }^{22}$ on the other hand, predicts a linear dependence, while Suzuki ${ }^{23}$ finds experimentally that for streamers in air $V_{S} \sim n_{1}^{1 / 2}$. Making use of the steadily increasing freeelectron density ahead of the streamer, we can, for a given set of conditions, determine the relationship between $V_{S}$ and $n_{1}$ for a single set of calculations such as shown in Fig. 2. We find that for a fixed applied voltage $V_{S}$ depends approximately logarithmically on $n_{1}$ over a voltage range of $125 \%$ to $175 \%$ of self-breakdown for streamers of both po- larities. Owing to the nature of this dependence, the data fit a power law nearly as well over this range. Writing $V_{S} \sim n_{1}^{\beta}, \beta$ varies from 0.6 to 0.3 for cathode-directed streamers and is nearly constant at 0.25 for anode-directed streamers as the applied voltage varies from $125 \%$ to $175 \%$ of self-breakdown. It must be pointed out, however, that true steady-state propagation has not been reached in these calculations. The propagation velocity is a strong function of the shape of the streamer head, which is slowly changing in time. Calculations carried out using different initial background electron densities also show an approximately logarithmic dependence of $V_{S}$ on $n_{1}$, but the value of $V_{S}$ for a given $n_{1}$ depends on the initial background density. The detailed dependence of $V_{S}$ on applied field is complex and will be presented elsewhere.

The algorithm we describe here can be applied to a very wide range of studies of streamers and other forms of ionizing waves. We have used the algorithm for a set of studies alluded to here which cover a range of applied fields from approximately the self-breakdown field $V_{\mathrm{SB}}$ to about $2 V_{\mathrm{SB}}$, and for a range of background preionization densities. This work is not presented in detail here, but will be discussed in a forthcoming publication. We have also used the algorithm for at least preliminary studies of the effects of more realistic photoionization mechanisms, the interaction between a focused laser beam and the propagating streamer head under conditions appropriate for experiments involving laser-triggered breakdown of spark gaps, and the effects of the shape of the streamer head. We have developed several methods for checking on the numerical accuracy of our algorithm, and have found it to behave well as long as the gradient of the electron density $n_{e}$ does not exceed the capabilities of the eighth-order flux calculator routine $(10 \%-90 \%$ rise in 1-2 grid points). Other areas of interest include guiding effects of channels of ionization or rarefication, propagation in nonuniform fields, other forms of ionizing waves such as return strokes, and various nonequilibrium effects.

This work was supported by the U.S. Air Force Office of Scientific Research Grant No. AFOSR-84-0032. We thank R. Morrow for originally pointing out the FCT algorithm to us, and the Texas Tech University Computing Center for providing computing time on a VAX $11 / 780$.
"Present address: Department of Electrical Science and System Engineering, Southern Illinois University, Carbondale, IL 62901.

${ }^{\dagger}$ Present address: Department of Electrical Engineering, University of Nebraska, Lincoln, NE 68588-0511.

${ }^{1}$ H. Raether, Z. Phys. 112, 464 (1939).

${ }^{2}$ L. B. Loeb and J. M. Meek, J. Appl. Phys. 11, 438 (1940).

3J. Koppitz, Z. Naturforsch. Teil A 26, 700 (1971).

${ }^{4}$ R. A. Dougal and P. F. Williams, J. Phys. D 17, 903 (1984).

${ }^{5}$ D. L. Turcotte and R. S. B. Ong, J. Plasma Phys. 2, 145 (1968).

${ }^{6}$ G. A. Shelton and R. G. Fowler, Phys. Fluids 11, 740 (1968).

${ }^{7}$ N. W. Albright and D. A. Tidman, Phys. Fluids 15, 86 (1972).

${ }^{8}$ R. Klingbeil, D. A. Tidman, and R. F. Fernsler, Phys. Fluids 15, 1969 (1972).

${ }^{9}$ A. J. Davies, C. S. Davies, and C. J. Evans, Proc. IEE 118, 816 (1971).

10L. E. Kline, J. Appl. Phys. 45, 2046 (1974).
${ }^{11}$ K. Yoshida and H. Tagashira, J. Phys. D 9, 491 (1976).

12I. Abbas and P. Bayle, J. Phys. D 13, 1055 (1980).

${ }^{13}$ R. Morrow, J. Comput. Phys. 43, 1 (1981).

14J. P. Boris and D. L. Book, J. Comput. Phys. 11, 39 (1973).

${ }^{15}$ S. T. Zalesak, J. Comput. Phys. 31, 335 (1979).

${ }^{16}$ R. Morrow and J. J. Lowke, J. Phys. D 14, 20 (1979).

${ }^{17}$ R. F. Fernsler, Ph.D. thesis, University of Maryland, 1975.

${ }^{18}$ E. E. Kunhardt and P. F. Williams, J. Comput. Phys. (to be published).

19J. Dutton, J. Phys. Chem. Ref. Data 4, 664 (1975).

${ }^{20}$ E. D. Lozanskii and D. B. Pontekorvo, Zh. Tekh. Fiz. 44, 2322

(1974) [Sov. Phys. Tech. Phys. 191432 (1975)].

${ }^{21}$ L. B. Loeb, Science 148, 1414 (1965).

22R. F. Fernsler, Phys. Fluids 27, 1005 (1984).

${ }^{23}$ T. Suzuki, J. Appl. Phys. 48, 5001 (1977). 

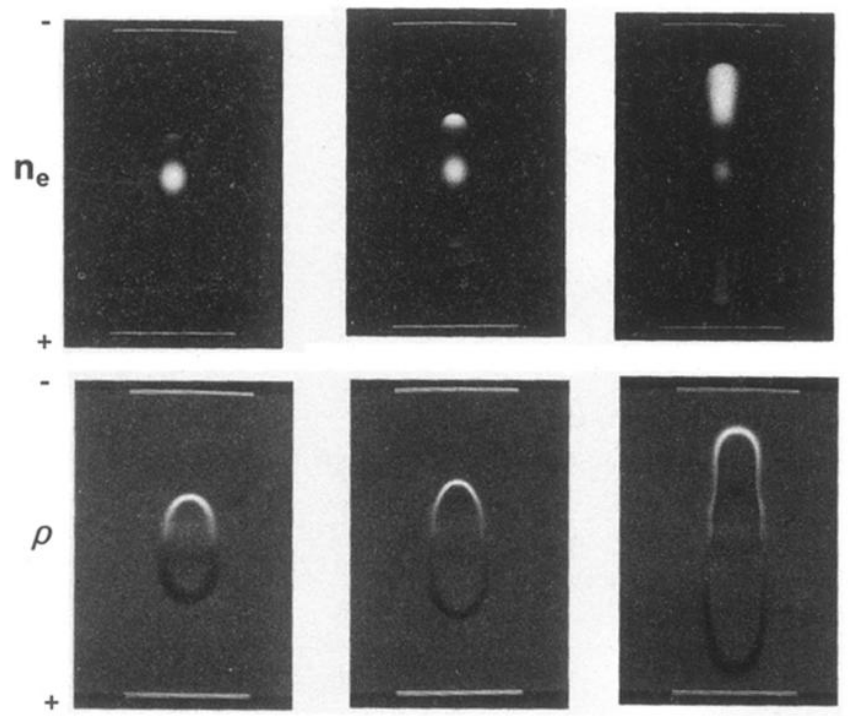

FIG. 1. Electron density $n_{e}$ and net charge density $\rho$, for $t=0.5$, 1.0 , and $1.25 \mathrm{~ns}$ for a calculation in which a Gaussian spheroid of plasma was initially placed in the center of the gap. The plasma density was $10^{14} \mathrm{~cm}^{-3}$ and the axial and transverse radii were 0.27 and $0.065 \mathrm{~mm}$, respectively. The gap spacing was $0.5 \mathrm{~cm}$, the voltage was $30 \mathrm{kV}$, making it about $70 \%$ overvolted, and there was a uniform background initial ionization density of $10^{8} \mathrm{~cm}^{-3}$. 